\title{
Estimation of Interfacial Shear in Ceramic Composites from Frictional Heating Measurements
}

\author{
Chongdu Cho, * John W. Holmes, \\ Ceramic Composites Research Laboratory, Department of Mechanical Engineering and \\ Applied Mechanics, The University of Michigan, Ann Arbor, Michigan 48109-2125
}

\begin{abstract}
A new approach for estimating the interfacial frictional shear stress in fiber-reinforced ceramics is presented. The approach is based upon measurement of the temperature rise which occurs during the cyclic loading of ceramic composites. This temperature rise, which is caused by the frictional slip of fibers within the composite, is related to the level of frictional shear stress which exists along debonded interfaces. To illustrate the technique, the interfacial shear stress in a unidirectional Nicalon-fiber calcium aluminosilicate matrix composite was determined at ambient temperature. [Key words: composite, interfaces, shear, friction, heating.]
\end{abstract}

\section{Introduction}

$\mathbf{T}$ THERE are currently several approaches used to determine the frictional shear stress which exists along the fiber/ matrix interface in fiber-reinforced ceramics. For example, Marshall ${ }^{1,2}$ developed a straightforward indentation technique (fiber pushout) that has gained widespread acceptance and has been used to determine the interfacial shear stress in polymeric-matrix, metal-matrix, and ceramic-matrix composites. A related approach, fiber pullout, ${ }^{3,4}$ has also been widely used to measure interfacial shear stress. Both fiber pushout and fiber pullout experiments provide information regarding the interfacial shear stress which would exist under monotonic loading conditions. To complement this information, there is a need to develop procedures which can be used to determinc the interfacial shcar stress which is present during fatigue loading.

Recent experiments by Holmes et al ${ }^{5-7}$ indicate that significant internal heating can occur during the cyclic loading of fiber-reinforced ceramics. For example, a temperature rise of approximately $100 \mathrm{~K}$ was observed during the roomtemperature fatigue of unidirectional SiC-fiber calcium aluminosilicate matrix composites at a frequency of $75 \mathrm{~Hz}$ and stress limits of $220 \mathrm{MPa}$ and $10 \mathrm{MPa}{ }^{7}$ The temperature rise which occurs during cyclic loading, as well as cyclic wear damage along the fiber/matrix interface, ${ }^{8}$ could lcad to differences in the interfacial shear stress which exists under monotonic and cyclic loading conditions. ${ }^{*}$ The primary mechanism of internal heating in fiber-reinforced ceramics involves the frictional slip of fibers along debonded fiber/matrix interfaces. For a frictional slip mechanism, the magnitude of

K.T. Habur - contributing cditor

Manuscript No. 196755. Received April 22, 1991; approved Augus1 2, 1991.

* Member, American Ceramic Society.

* Under cyclic loading, differential thermal expansion between the fiber and matrix could lead to a frequency dependence of frictional shear stress (note that the degree of differential thermal expansion is determined by the thermal expansion mismatch between the fiber and matrix and the temperathermal expansion mismatch between the fiber and matrix and the tempera-
ture rise which occurs during fatigue; the temperature rise is strongly influenced by both loading frequency and applied stress range ${ }^{5}$ ). internal heating would be related to the average frictional shear stress which exists along the fiber/matrix interface. Thus, by equating the work performed in the frictional sliding of fibers to the heat energy dissipated by a fatigue specimen, it should be possible to estimate the average level of frictional shear stress present along the fiber/matrix interface; the development of this approach forms the basis of the present papcr.

\section{Analysis}

\section{(I) Work Performed in the Frictional Slip of Fibers}

It is assumed that the cyclic frictional sliding of unfractured fibers within debonded interfacial slip zones is the primary mechanism of internal heating which occurs in fibcr-rcinforced ceramics. The distance over which interfacial slip occurs during cyclic loading is controlled by the magnitude of the external load and the direction of loading,2,10,11 thus, the active slip length changes throughout a fatigue cycle. Depending upon the loading history, temperature, and composite system, fibers can undergo either partial slip or a combination of partial and full frictional slip. Thus for generality, two cases must be considered when determining the work performed in the frictional sliding of fibers: (I) partial frictional slip and, (II) partial/full frictional slip. In the analysis which follows, the slip zone formed during loading will be referred to as a forward slip zone; the slip zone formed during unloading is referred to as a reverse slip zone.

\section{(A) Case I: Partial Frictional Slip}

For purposes of discussion, it is assumed that a composite has been subjected to the loading history shown in Fig. 1. A specimen is first loaded from zcro stress to a stress $\sigma_{\text {max }}$ along path $\mathrm{OA}^{\prime}$. During this initial loading, matrix cracking and interfacial debonding occur; this leads to the formation of a forward slip zone of length $l_{\text {fwd }}$ (Fig. 2). For path OA', the length of the forward slip zone is equal to the distance over which debonding occurs. Neglecting elastic deformation outside the slip zones, the fiber debond length $\delta$ can be estimated using an equation similar to that given by Thouless and Evans ${ }^{12}$ for monotonic loading. At the maximum fatigue stress, $\sigma_{\text {max }}$, the forward slip-zone length is ${ }^{*}$

$$
l_{\text {iwd }}\left(\sigma_{\text {max }}\right)=\delta=\frac{C d_{f} \sigma_{\text {max }}}{4 \tau_{\lrcorner}}
$$

where, $\tau_{d}$ is the dynamic shear stress (frictional) along the fiber/matrix interface, ${ }^{\S} d_{\mathbb{f}}$ is the fiber diameter, and $C$ is given

Partial slip refers to the situation where the active interfacial slip length is always less than one-half' of the matrix crack spacing. Full slip occurs when the active slip length equals one-hall of the matrix crack spacing.

Note that the expression given by Thouless and Evans" for slip lenuth is given in terms of the fiber stress, whereas Eq. (1) is expressed in terms of the far-field stress.

"The terminology "dynamic shear stress" is used to emphasize that the frictional shear stress which is measured under cyclic loading conditions may differ from that measured using fiber pushout or pullout experiments. 


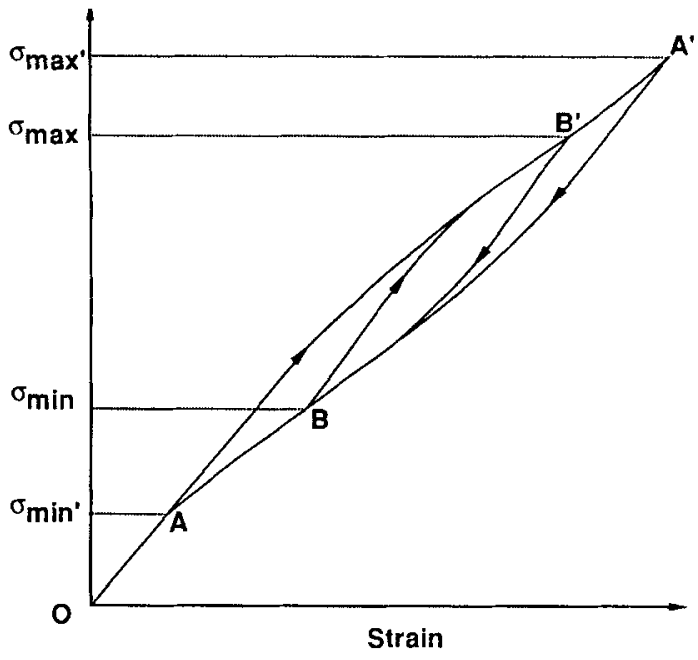

Fig. 1. Loading path used to calculate the frictional energy dissipation in fiber-reinforced ceramics subjected to cyclic loading. Consistent with the experiments used to obtain a stable crack density prior to temperature rise measurements, initial fatigue is assumed to occur between stress limits of $\sigma_{\max }$ and $\sigma_{\min }$ (path $\mathrm{AA}^{\prime}$ ). Subsequent fatigue cycles occur at a lower stress range, between stress limits of $\sigma_{\max }$ and $\sigma_{\min }\left(\right.$ path $\left.\mathrm{BB}^{\prime}\right)$.

in terms of the volume fraction of fibers $\left(v_{\mathrm{f}}\right)$ and the tensile moduli of the matrix and composite $\left(E_{\mathrm{m}}\right.$ and $E_{\mathrm{c}}$, respectively):

$$
C=\frac{\left(1-v_{\mathrm{f}}\right) E_{\mathrm{m}}}{v_{\mathrm{f}} E_{\mathrm{c}}}
$$

Upon stress reversal at point $\mathrm{A}^{\prime}$, the loading path $\mathrm{A}^{\prime} \mathrm{A}$ is followed to the minimum cycle stress $\sigma_{\text {min' }}$. After achieving a stable crack density characteristic of fatigue at a maximum stress $\sigma_{\max }$, subsequent fatigue cycles are assumed to occur along path $\mathrm{BB}^{\prime}\left(\sigma_{\max } \leq \sigma_{\max }\right)$. The first loading path $\mathrm{OA}^{\prime} \mathrm{A}$ was included for generality since it determines the residual stress state which exists during subsequent loading at lower peak stresses. The instantaneous slip-zone length $l_{\text {fwd }}(\sigma)$ formed during loading from $\sigma_{\min }$ to $\sigma$ can be found by graphical analysis of the matrix stress distribution shown in Fig. 3:

$$
l_{\mathrm{fwd}}(\sigma)=\frac{C d_{\mathrm{f}}}{8 \tau_{\mathrm{d}}}\left(\sigma-\sigma_{\min }\right) \quad\left(\sigma_{\min } \leq \sigma \leq \sigma_{\max }\right)
$$

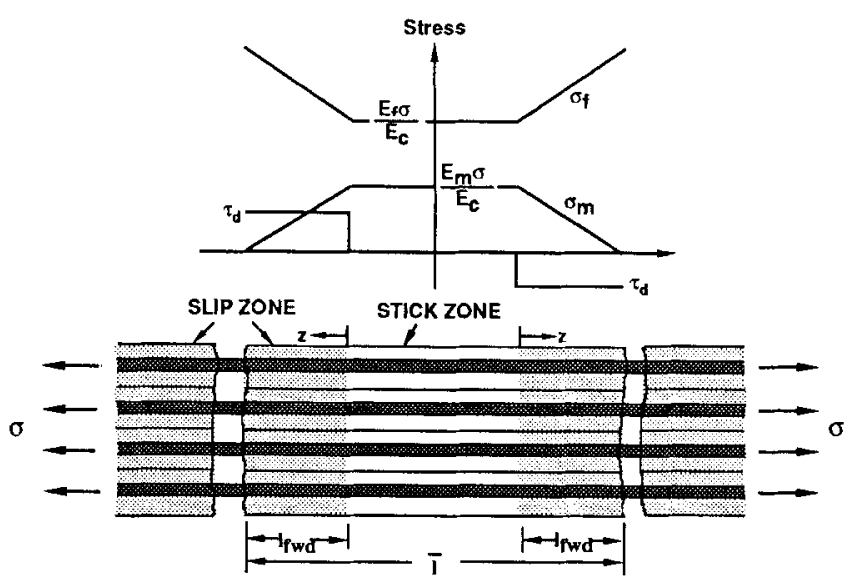

Fig. 2. Axial stress distribution in the matrix and fiber during initial monotonic loading from zero stress to an intermediate stress level $\sigma$. As a simplification, the shear stress is assumed to be constant within the interfacial slip zones of length $l_{\text {fwd }}$ (an exact solution for the stress distribution can be found in Ref. 9). The unit cell has a length equal to the experimentally determined mean crack spacing.
Outside the slip zones, there is no relative displacement between the fiber and matrix; these regions of "stick" do not enter into the calculations for frictional work. The changes in fiber and matrix stress along the interface which occur during the loading portion of the fatigue cycle can be determincd by graphical analysis of the residual (prior) and current stress states shown in Fig. 3. For a change in stress from $\sigma_{\min }$ to $\sigma$, the stress increments can be cxpressed as follows:

$$
\begin{aligned}
& \delta \sigma_{\mathrm{f}}(z)=\frac{E_{\mathrm{f}}}{E_{\mathrm{c}}}\left(\sigma-\sigma_{\min }\right)+\frac{8 \tau_{\mathrm{d}} z}{d_{\mathrm{l}}} \quad\left(0<z<l_{\text {iwd }}\right) \\
& \delta \sigma_{\mathrm{m}}(z)=\frac{E_{\mathrm{m}}}{E_{\mathrm{c}}}\left(\sigma-\sigma_{\mathrm{min}}\right)\left(1-\frac{z}{l_{\mathrm{fwd}}}\right) \quad\left(0<z<l_{\mathrm{fwd}}\right)
\end{aligned}
$$

The corresponding increments in fiber and matrix displacement along the interface (denoted by $\delta l_{\mathrm{m}}$ and $\delta l_{f}$, respectively) can be obtained by integration of the stress increments:

$$
\begin{aligned}
& \delta l_{\mathrm{i}}(z)=\frac{\left(\sigma-\sigma_{\min }\right) z}{E_{\mathrm{c}}}+\frac{4 \tau_{\mathrm{d}} z^{2}}{d_{\mathrm{f}} E_{\mathrm{f}}} \\
& \delta l_{\mathrm{m}}(z)=\frac{\left(\sigma-\sigma_{\min }\right)}{E_{\mathrm{c}}}\left(z-\frac{z^{2}}{2 l_{\mathrm{fwd}}}\right)
\end{aligned}
$$

The relative incremental displacement between the fiber and matrix, $\delta l_{\text {slide }}$, is given by the difference between the fiber and matrix displacements (Eqs. (6) and (7)):

$$
\delta l_{\text {slide }}(z)=\delta l_{\mathrm{f}}(z)-\delta l_{\mathrm{m}}(z)=\frac{4 \tau_{\mathrm{d}} z^{2}}{C d_{\mathrm{f}} v_{\mathrm{f}} E_{\mathrm{f}}}
$$

(Note that Eq. (3) was substituted for $l_{\text {fwd }}$ when simplifying Eq. (8).) The increment in frictional work performed during loading is given by the incremental sliding distance $\left(\delta l_{\text {slide }}\right)$ multiplied by the frictional force $\left(\pi d_{\uparrow} \tau_{\mathrm{d}} \mathrm{d} z\right)$. Using an approach similar to that discussed by Thouless ${ }^{13}$ for monotonic loading, the frictional work performed during loading from $\sigma_{\min }$ to $\sigma_{\max }$ can be determined by integrating over the active slip length:

$$
W_{\text {load }}=2 \int_{0}^{l_{\mathrm{fwd}}\left(\sigma_{\mathrm{mlax}}\right)} \delta l_{\text {slide }}(z)\left[\pi d_{\mathrm{l}} \tau_{\mathrm{d}}\right] \mathrm{d} z=\frac{\pi d_{\mathrm{f}}^{3} C^{2} \Delta \sigma^{3}}{(3)(64) v_{\mathrm{f}} E_{\mathrm{f}} \tau_{\mathrm{d}}}
$$

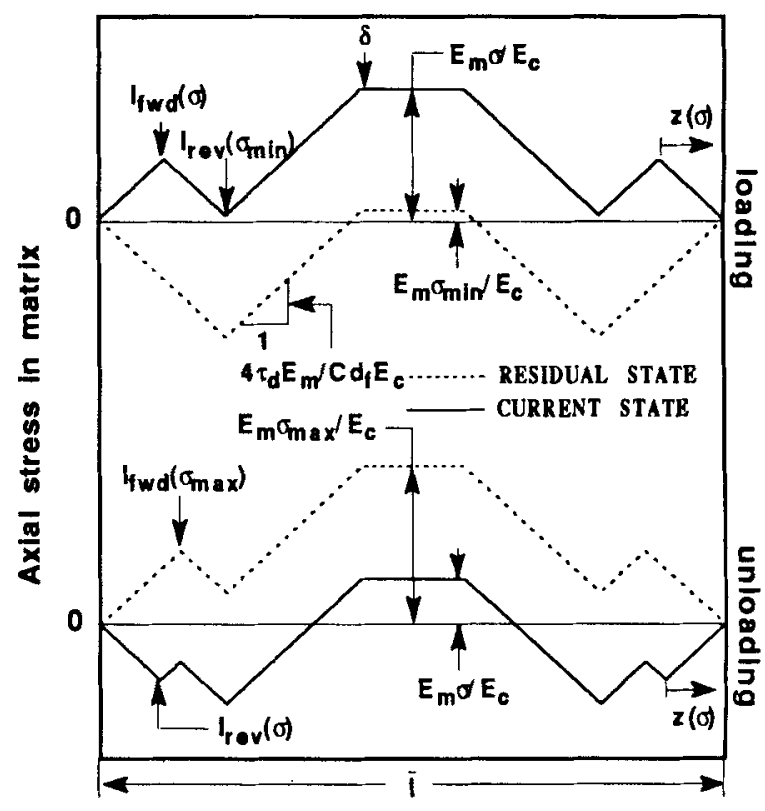

Axlal distance

Fig. 3. Stress distribution in the matrix during loading and unloading for the case of partial frictional slip. In the figure shown, loading is assumed to occur from $\sigma_{\min }$ to an intermediate stress $\sigma$, with unloading occurring from $\sigma_{\text {max }}$ to $\sigma$. 
where $\Delta \sigma=\sigma_{\max }-\sigma_{\text {min }}$, and the factor of two is used to account for symmetry of the interfacial slip zones (see Fig. 2).

Upon unloading from $\sigma_{\max }$ to $\sigma$, a reversed slip zone of length $l_{\text {rev }}(\sigma)$ develops:

$$
l_{\mathrm{rcv}}(\sigma)=\frac{C d_{r}}{8 \tau_{\mathrm{d}}}\left(\sigma_{\mathrm{max}}-\sigma\right) \quad\left(\sigma_{\min } \leq \sigma \leq \sigma_{\operatorname{mix}}\right)
$$

The frictional work performed during unloading can be shown to be equal to that for loading (this can be shown using an analysis similar to that used to derive Eq. (9)). Thus, the total work performed in one complete loading-unloading cycle becomes

$$
W_{\text {total }}=2 W_{\text {load }}=2\left(\frac{\pi d_{\mathrm{f}}^{3} C^{2} \Delta \sigma^{3}}{(3)(64) v_{\mathrm{f}} E_{\mathrm{f}} \tau_{\mathrm{d}}}\right)=\frac{\pi d_{\mathrm{f}}^{3} C^{2} \Delta \sigma^{3}}{96 v_{\mathrm{l}} E_{\mathrm{l}} \tau_{\downarrow}}
$$

Dividing Eq. (11) by the unit cell volume, $\pi d_{l}^{2} \bar{l} / 4 v_{\mathrm{f}}$ (see Fig. 2), and multiplying by the loading frequency $f$, gives the total frictional encrgy dissipation per unit volume:

$$
\frac{\mathrm{d} w_{\text {tric }}}{\mathrm{d} t}=\frac{f C^{2} d_{\mathrm{i}} \Delta \sigma^{3}}{24 E_{\mathrm{i}} \bar{l} \tau_{\mathrm{u}}}
$$

Note that the above analysis holds even if full frictional slip occurs during initial loading to $\sigma_{\text {max }}$ (path $\mathrm{OA}^{\prime}$ ); the only requirement is that full slip does not occur during subsequent loading between $\sigma_{\text {max }}$ and $\sigma_{\text {min }}$ (path $\mathrm{BB}^{\prime}$ ). The case where both partial and full frictional slip occur within the same fatigue cycle is analyzed below.

\section{(B) Case II: Partial/Full Frictional Slip}

As with case I (partial frictional slip), the specimen is first loaded from zero stress to a stress $\sigma_{\text {max }}\left(\right.$ path OA'). If $\sigma_{\text {max }}$ is of sufficicnt magnitude, the interfacial slip zones will become saturated at an intermediate level of stress denoted by $\sigma_{\text {slip }}$ (i.c., at $\sigma_{\text {slip }}$, interfacial slip occurs over a distance equal to the mean crack spacing $\bar{l}$ ). During initial loading along path $\mathrm{OA}^{\prime}$, the applied stress level at which full frictional slip begins is determined by the interfacial shear stress and mean crack spacing $\bar{l}$ :

$$
\sigma_{\text {slip }}=\frac{2 \tau_{\mathrm{d}} \bar{l}}{C d_{1}}
$$

Upon unloading from $\sigma_{\max }$ to an intermediate stress $\sigma$, full frictional slip will begin when the change in stress equals $2 \sigma_{\text {slip. }}$. After unloading to $\sigma_{\min }$ (path $\mathrm{A}^{\prime} \mathrm{B}$ ), the residual stress state shown in Fig. 4 will exist. Reloading from $\sigma_{\min }$ to an intermediate stress $\sigma$ gives rise to a forward slip zone of length $I_{\mathrm{fwd}}(\sigma)$ (see Fig. 4):

$$
l_{\mathrm{fwd}}(\sigma)=\frac{C d_{\mathrm{r}}}{8 \tau_{\mathrm{d}}}\left(\sigma-\sigma_{\mathrm{min}}\right)
$$

If the stress range during subsequent loading to $\sigma_{\max } \mathrm{ex}$ ceeds $2 \sigma_{\text {slip }}$, full frictional slip occurs. As the stress is increased beyond that ncccssary to initiate full slip, the additional increment in external load is supported only by the fibers. To determine the work performed in the frictional slip of fibers, the loading path must be divided into two regimes: (a) a partial slip regime which occurs for $\sigma_{\min }<\sigma<\sigma_{\min }+$ $2 \sigma_{\text {slip }}$ and, (b) a full slip regime which occurs for $\sigma_{\min }+$ $2 \sigma_{\text {slip }}<\sigma<\sigma_{\max }$. Since the work performed during loading and unloading is equal, only the work performed during loading is considered in the analysis which follows.

(a) Partial Slip: $\sigma_{m i n}<\sigma<\sigma_{\min }+2 \sigma_{\text {slip }}$. Within this interval, the analysis is similar to case $I$. The changes in fiber and matrix stress along the interface which occur during the loading portion of the fatigue cycle can be determined by graphical analysis of the residual (prior) and current stress states shown in Fig. 4. As with case I (Eq. (9)), the stress increments can be used to determine the relative incremental displacement between the fiber and matrix, $\delta l_{\text {sliste, }}$ which can

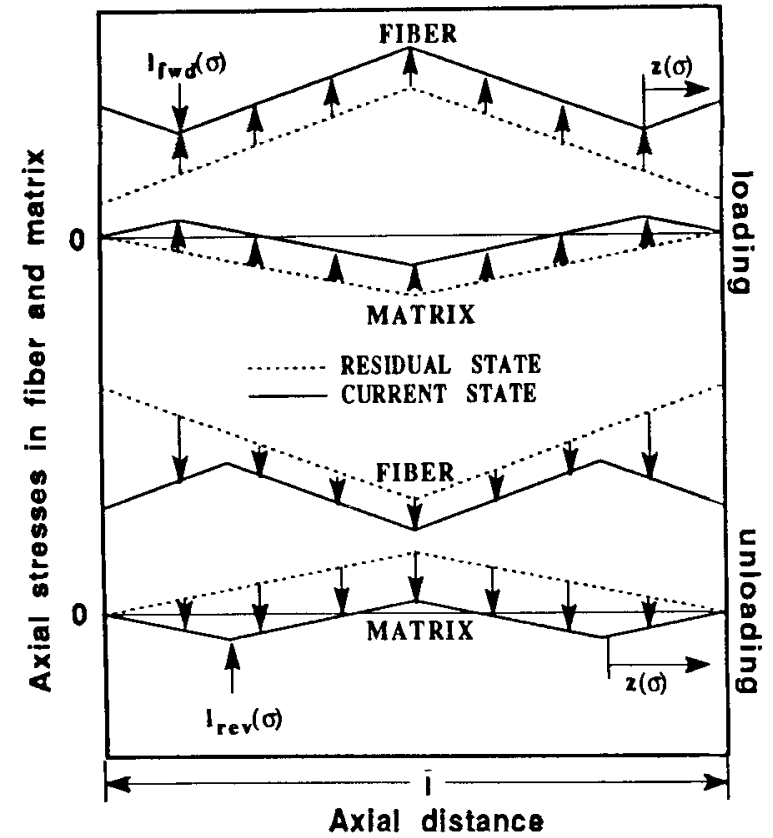

Fig. 4. Stress distribution in the fiber and matrix during loading and unloading for the case of partial/full frictional slip. The figure shown was used to determine the frictional work performed during the partial slip portions of the fatigue cycle. Partial frictional slip occurs during the initial stages of loading or unloading; for this stage of loading, only those regions where $z \geq 0$ contribute to the frictional work. When full frictional slip is reached during loading or unloading, the origin of $z(\sigma)$ moves to $l / 2$, i.e., slip occurs throughout a slip zone.

then be integrated to determine an expression for the frictional work performed during loading.

$$
W_{\text {load }}=\frac{\pi d_{1}^{3} C^{2}\left(2 \sigma_{\mathrm{stip}}\right)^{3}}{(3)(64) v_{\mathrm{f}} E_{\mathrm{f}} \tau_{\mathrm{d}}}
$$

(b) Full Slip: $\sigma_{m i n}+2 \sigma_{\text {sip }}<\sigma<\sigma_{\max }$ : In this interval, the forward slip length, $l_{\text {fwd }}$, equals one-half of the mean crack spacing, $\bar{l}$. As noted above, once full frictional slip occurs, the additional load is supported entirely by the fibers; no further change in matrix stress occurs. The incremental changes in axial stress in the fiber and matrix which occur during loading are

$$
\begin{aligned}
& \delta \sigma_{\mathrm{m}}(z)=0 \\
& \delta \sigma_{\mathrm{I}}(z)=\frac{\sigma_{\text {max }}-\left(\sigma_{\mathrm{min}}+2 \sigma_{\mathrm{slip}}\right)}{v_{\mathrm{i}}}=\frac{\Delta \sigma-2 \sigma_{\text {slip }}}{v_{\mathrm{f}}}
\end{aligned}
$$

Since only the fibers undergo additional loading, the strain increment $\left(\delta \sigma_{\mathrm{f}}(z) / E_{\mathrm{l}}\right)$ is constant within a slip zonc. When this condition is reached, the relative incremental displacement between the fiber and matrix at a distance $z$ from the edge of the slip zone, $\delta l_{\text {slide }}(z)$, equals the increment in fiber displacement, $\delta l_{\uparrow}(z)$ :

$$
\delta l_{\text {slide }}(z)=\delta l_{\mathrm{f}}(z)=\frac{\left(\Delta \sigma-2 \sigma_{\mathrm{slip}}\right) z}{v_{\mathrm{f}} E_{\mathrm{f}}}
$$

Within the range $\sigma_{\min }+2 \sigma_{\text {slip }}<\sigma<\sigma_{\max }$, the frictional work performed during loading to $\sigma_{\max }$ can be obtained by integrating Eq. (18) over the active slip length (i.e., from $z=0$ to $z=l / 2)$ :

$$
\begin{aligned}
W_{\text {load }} & =2 \int_{0}^{\bar{l} 2} \delta l_{\text {slide }}(z)\left[\pi d_{\mathrm{f}} \tau_{\mathrm{d}}\right] \mathrm{d} z \\
& =\left(\frac{\pi d_{\mathrm{f}}^{2} l}{4 v_{\mathrm{f}}}\right)\left(\frac{\bar{l} \tau_{\mathrm{d}}}{d_{\mathrm{f}}}\right)\left(\frac{\Delta \sigma-2 \sigma_{\text {slip }}}{E_{\mathrm{l}}}\right)
\end{aligned}
$$


Adding Eqs. (15) and (19), the total frictional work performed during loading from $\sigma_{\min }$ to $\sigma_{\max }$ can be cxpressed as

$$
W_{\text {load }}=\frac{\pi d_{\mathrm{f}}^{2} \bar{l}}{4 v_{\mathrm{f}}}\left[\frac{d_{\mathrm{f}} C^{2}\left(2 \sigma_{\mathrm{sip}}\right)^{3}}{(3)(16) E_{\mathrm{f}} \bar{l} \tau_{\mathrm{d}}}+\left(\frac{\bar{l}_{\tau_{\mathrm{d}}}}{d_{\mathrm{f}}}\right)\left(\frac{\Delta \sigma-2 \sigma_{\mathrm{silp}}}{E_{\mathrm{f}}}\right)\right]
$$

As noted earlier, the frictional work performed during loading and unloading is equal; thus, $\boldsymbol{W}_{\text {total }}=2 \boldsymbol{W}_{\text {load }}$. Substituting $4 \tau_{\mathrm{d}} \vec{l} / C d_{\mathrm{f}}$ for $2 \sigma_{\mathrm{slip}}$ in $\mathrm{Eq}$. (20), dividing by the unit cell volume, and multiplying by the loading frequency $f$, gives the total frictional work rate when both partial and full frictional slip occur within a fatigue cycle:

$$
\frac{\mathrm{d} w_{\text {rric }}}{\mathrm{d} t}=\frac{2 f \bar{l} \tau_{\mathrm{d}} \Delta \sigma}{d_{\mathrm{i}} E_{\mathrm{l}}}-\frac{16 f \tau_{\mathrm{d}}^{2} \bar{l}^{2}}{3 E_{\mathrm{f}} C d_{\mathrm{l}}^{2}}
$$

\section{(2) Calculation of Heat Loss During Fatigue}

Assuming that a steady-state microstructural damage state and temperature rise have been attained in a composite during cyclic loading, and neglecting other modes of energy dissipation, the heat loss from the composite to isothermal surroundings must equal the work performed in the frictional slip of fibers. For a fatigue specimen, such as that shown in Fig. 5, convective and radiative heat losses $\left([\mathrm{d} q / \mathrm{d} t]_{\text {conv }}\right.$ and $[\mathrm{d} q / \mathrm{d} t]_{\mathrm{rad}}$, respectively) will occur from the specimen surface (the surface area is denoted by $A_{\text {sur }}$ ). Additional heat loss by conduction $\left([\mathrm{d} q / \mathrm{d} t]_{\text {cind }}\right)$ will occur through a cross section which is perpendicular to the fibers (denoted by $A_{\text {cond }}$ ). The total rate of heat loss per unit volume, $\mathrm{d} q / \mathrm{d} t$, is given in terms of the surface temperature of the specimen $T_{\mathrm{s}}$, air temperature $T_{\mathrm{a}}$, convective heat transfer coefficient $h$, cmissivity $\varepsilon$, and the thermal conductivity of the composite parallel to the fibers $k: 1$

$$
\begin{aligned}
\frac{\mathrm{d} q}{\mathrm{~d} t}= & {\left[\frac{\mathrm{d} q}{\mathrm{~d} t}\right]_{\mathrm{conv}}+\left[\frac{\mathrm{d} q}{\mathrm{~d} t}\right]_{\mathrm{rad}}+\left[\frac{\mathrm{d} q}{\mathrm{~d} t}\right]_{\text {cond }}=} \\
& {\left[h\left(T_{\mathrm{s}}-T_{\mathrm{a}}\right)+\varepsilon \beta\left(T_{\mathrm{s}}^{4}-T_{\mathrm{a}}^{4}\right)\right] \frac{A_{\text {surf }}}{V} } \\
& +\frac{2 k A_{\text {cond }}}{V}\left[\frac{\Delta T}{\Delta z}\right]_{\text {axial }}
\end{aligned}
$$

where $\beta$ is the Stefan-Boltzman constant $(5.67 \times$ $\left.10^{-8} \mathrm{~W} / \mathrm{m}^{2} \cdot \mathrm{K}^{4}\right),[\Delta T / \Delta z]_{\text {axial }}$ is the axial temperature gradient at the end of the gage section, and $V$ is the gage-section volume. It is important to note that as an alternative to estimating $\mathrm{d} q / \mathrm{d} t$ from Eq. (22) (which requires measurement of the temperature rise, $\left(T_{s}-T_{\mathrm{a}}\right)$, the energy dissipation during cyclic loading can be estimated by calculating the area enclosed by a cyclic stress-strain (hysteresis) curve and multiplying by the loading frequency $f$ (this also provides a means by which the accuracy of the heat loss analysis can be determined). Under stcady-state conditions, the mechanical work performed in the frictional slip of fibers $\mathrm{d} w_{\text {fric }} / \mathrm{d} t$ (Eq. (12) or (21)) must equal the rate of heat loss to the surroundings $\mathrm{d} q / \mathrm{d} t$ (Eq. (22)). Thus, equating $\mathrm{d} w_{\text {fric }} / \mathrm{d} t$ to $\mathrm{d} q / \mathrm{d} t$ (or alternatively equating $\mathrm{d} w_{\text {tric }} / \mathrm{d} t$ to the energy dissipation calculated from cyclic stress-strain curves) provides an equation where the only unk nown is the frictional shear, $\tau_{\mathrm{d}}$.

\section{Application of Model}

To illustrate the application of the analysis discussed above, the frictional shear stress present in a unidirectional Nicalon/CAS-1I** composite (Nippon Carbon, Tokyo, Japan, and Corning Inc, Corning, NY, respectively) will be estimated. The technique involves conducting tension-tension

\footnotetext{
"For a general discussion of heat transfer the reader is relerred to Holman.

${ }^{*}$ CAS-II is the Corning designation for a calcium aluminosilicate matrix.
}
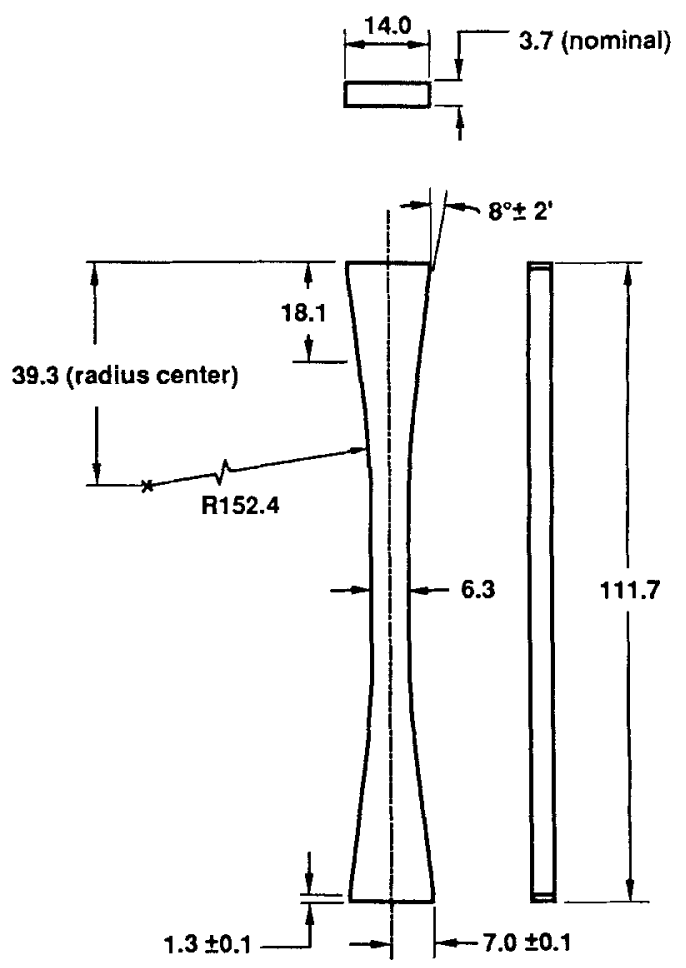

Fig. 5. Edge-loaded specimen used in the fatigue experiments The specimen had a gage length of $33 \mathrm{~mm}$. Other specimen geometries and gripping arrangements can be utilized so long as a large volume of uniformly stressed material is present (e.g., faceloaded rectangular specimens). Dimensions a re in millimeters.

fatigue experiments and determining the rate of heat loss from the specimen. Prior to conducting the fatigue experiments, it is important that a stable matrix crack spacing has been achicved; this is most easily accomplished by prefatiguing a specimen at a maximum stress which is equal to or greater than the peak stress used in the subsequent frictional heating experiments.

The 16-ply composite was processed with 35 vol\% fibers. Edge-loaded tensile specimens, with a $33-\mathrm{mm}$ gage length, were used in the fatigue experiments (Fig. 5). The experiments were performed in air using an isothermal test chamber which completely surrounded the specimen and grips. ${ }^{7}$ The chamber was held at a temperature of $293 \mathrm{~K} \pm 0.1 \mathrm{~K}$; the relative humidity within the chamber was $65 \%$. All fatigue cxperiments werc conducted at a sinusoidal loading frequency of $25 \mathrm{~Hz}$. The temperature rise at the specimen surface was measured using a high-resolution infrared pyrometer (Model No. 5402, Everest Interscience, Fullerton, CA) which was focused at the center of the specimen gage section. The cyclic stress-strain curves were also recorded to allow comparing the frictional energy dissipation with $\mathrm{d} q / \mathrm{d} t$ (Eq. (22)). Further details concerning the experimental procedure can be found in Refs. 6 and 7.

To achicve a constant matrix crack spacing prior to measurement of the temperature rise, a specimen was first prefatigued for 100000 cycles between fixed stress limits of 180 and $10 \mathrm{MPa}$ (Fig. 6(A)). ${ }^{\dagger \dagger}$ After approximately $20000 \mathrm{cyclcs}$, the mean crack spacing approached a platcau at approximately $198 \mu \mathrm{m}$ (the crack spacing was determined by periodically unloading the specimen and using surface replication with acctate film). Next, as illustrated in Fig. 6(A), the specimen was subjected to cyclic loading at stress ranges of 90,110 , and $130 \mathrm{MPa}$. Several stress ranges were included to determine the sensitivity of the technique to peak stress and stress range

${ }^{i} \Lambda$ peak stress below the proportional limit strength (225 MPa (Ref. 6)) was chosen to minimize fiber fracture. 


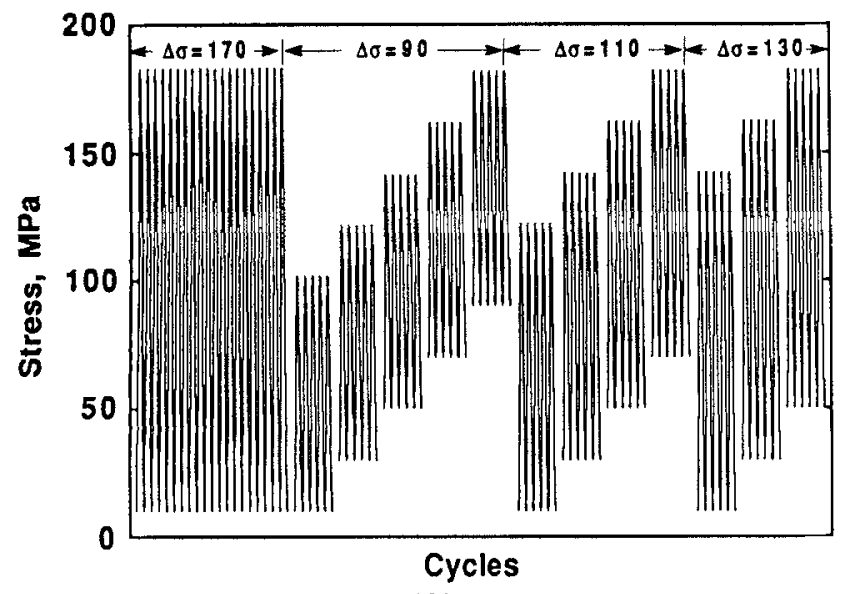

(A)

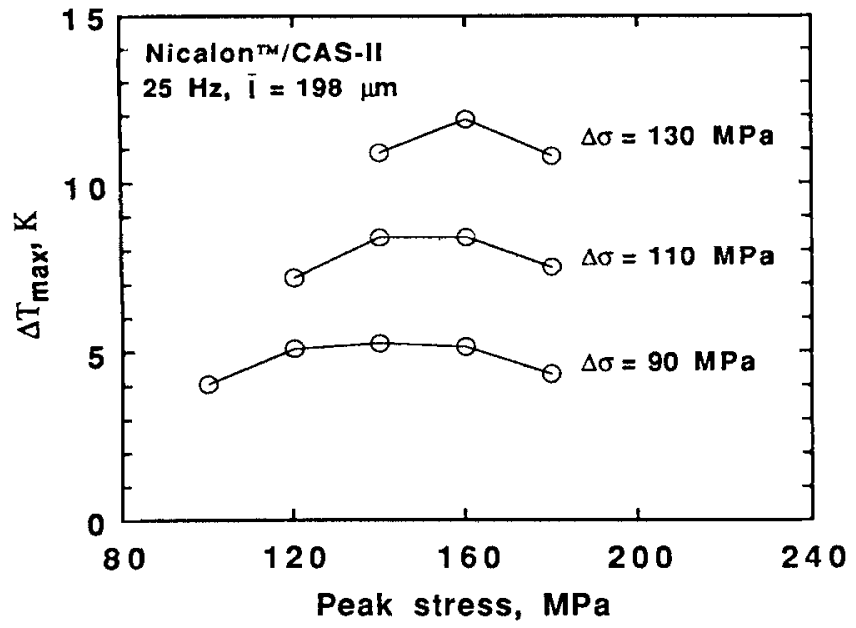

(B)

Fig. 6. (A) Loading history used to determine the influence of stress range on temperature rise. To achieve a stable crack density, the specimen was initially fatigued between stress limits of 180 and $10 \mathrm{MPa}$. Subsequent fatigue experiments were conducted for 25000 cycles each. All experiments were conducted at a loading frequency of $25 \mathrm{~Hz}$. The stress range (in MPa) is given above each group of stress-cycle curves. (B) Temperature rise versus stress range for unidirectional Nicalon/CAS-II. The initial temperature of the composite was $293 \mathrm{~K}$.

and to provide confidence in the data trends. Since the amount of energy dissipation is influenced by matrix crack spacing, it is important that the maximum stress used in the prefatigue experiment is not exceeded. The steady-state temperature rise measured for each stress range is shown in Fig. 6(B). Measurements of the average crack spacing, which were made after each constant stress-range experiment, showed that the crack spacing remained essentially constant at $198 \mu \mathrm{m}$.

Using the data given in Table I and the temperature rise data given in Fig. 6(B), the dynamic shear stress was determined for the two frictional work cases discussed above - i.e., partial slip and partial/full frictional slip. Assuming partial frictional slip, and using Eqs. (12) and (22) for the energy balance, $\tau_{\downarrow}$ would be between 3.5 and $5.0 \mathrm{MPa}$ for the three stress ranges examined (see Fig. 7). Assuming that partial/full frictional slip occurs during fatigue, and using Eqs. (21) and (22), $\tau_{\mathrm{d}}$ would be approximately $0.4 \mathrm{MPa}$ for all stress ranges. Experiments conducted on two additional specimens gave essentially the same values for frictional shear stress (the values differed by less than 5\%). As mentioned above, as an alternative to calculating the rate of heat loss from Eq. (22), the energy dissipation can be determined from the cyclic stressstrain curves and equated directly to Eqs. (12) or (21). Using this approach provided results which were similar to those obtained by using the heat loss equation (the comparison for the case of partial frictional slip is shown in Fig. 7).

To determine which analysis (i.e., partial slip or partial/full slip) was applicable for the temperature and loading conditions which were examined, the experimentally measured cyclic stress-strain curves (Fig. 8(A)) were compared with those predicted using the values of frictional shear stress calculated above. To calculate the cyclic stress-strain curves, the stress and strain distribution in the fiber was estimated for the cases of partial slip and partial/full slip (a graphical approach similar to that shown in Figs. 3 and 4 was used to determine the stress-strain curves). ${ }^{\ddagger \neq}$ Figure $8(\mathrm{~B})$ shows the calculated cyclic stress-strain curves for the partial slip assumption. Although the predicted modulus was much higher than the experimentally measured modulus, the dependence of average modulus on stress range showed a similar trend (i.e., in both instances, the average modulus decreased as the maximum fatigue stress was increased). ${ }^{85}$ This trend is consistent with partial slip behavior. Assuming partial/full slip occurs during the fatigue cycle $\left(\tau_{\mathrm{d}}=0.4\right)$, the average modulus would remain constant as the maximum fatigue stress is increased; this result is not consistent with the experimentally observed trend shown in Fig. 8(A). For the case of partial slip, the discrepancy between the calculated modulus and the experimentally measured modulus is thought to arise from fiber fracture which occurred during the initial fatigue experiments which were conducted between stress limits of 10 and $180 \mathrm{MPa}$ (note that fiber fracture was not included when de-

\footnotetext{
FFrom the axial stress distribution in the fiber, the axial strain in the slip zones is found directly through integration of the displacement. Within the regions bridged by fibers, the additional strain resulting from fiber loading is given by $\sigma / \nu_{\mathrm{f}} E_{\mathrm{f}}$.

The decrease in modulus is caused by the increase in the slip-zone length which occurs as the applied stress is increased.
}

Table I. Data Used to Estimate $\tau_{d}$ for a Unidirectional 16-Ply Nicalon/CAS-II Composite

\begin{tabular}{|c|c|}
\hline Parameter & Comments \\
\hline $\begin{array}{l}A_{\text {surf }} \text { (surface area of specimen gage section) }=662.6 \mathrm{~mm}^{2} \\
A_{\text {cond }} \text { (cross-sectional area of specimen gage section) }=23.3 \mathrm{~mm}^{2} \\
E_{\mathrm{r}} \text { (tensile modulus of fiber) }=200 \mathrm{GPa} \\
E_{\mathrm{n}} \text { (tensile modulus of matrix) }=88 \mathrm{GPa} \\
f \text { (loading frequency used in fatigue experiments) }=25 \mathrm{~Hz} \\
h \text { (free convection heat transfer coefficient) } \\
k \text { (thermal conductivity of composite parallel to the fibers) }=5.16 \mathrm{~W} / \mathrm{m} \cdot \mathrm{k} \\
l \text { (mean crack spacing) }=198 \mu \mathrm{m} \\
v_{\mathrm{f}} \text { (fiber volume fraction) }=0.35 \\
\Delta T \text { (steady-state temperature rise) } \\
{[\Delta T / \Delta z]_{\text {axial }} \text { (average axial temperature gradient from end of gage scction to grips) }} \\
V \text { (gage section volume) }=768.9 \mathrm{~mm}^{3} \\
\varepsilon \text { (surface emissivity of composite) }=1.0\end{array}$ & $\begin{array}{l}\text { Fig. } 5 \\
\text { Fig. } 5 \\
\text { Ref. } 14 \\
\text { Ref. } 14 \\
\text { Ref. } 15^{\star} \\
\text { Ref. } 16 \\
\text { From microscopy } \\
\text { From microscopy } \\
\text { Fig. } 6 \text { (B) } \\
\text { Fig. } 5 \\
\text { Assumed value }\end{array}$ \\
\hline
\end{tabular}

$\star h=1.42\left(T_{\mathrm{x}}-T_{\mathrm{i}} / L\right)^{1 / 4}\left[\right.$ units: $\left.\mathrm{W} / \mathrm{m}^{2} \cdot \mathrm{K}\right] . L$ refers to the gage-section length. 


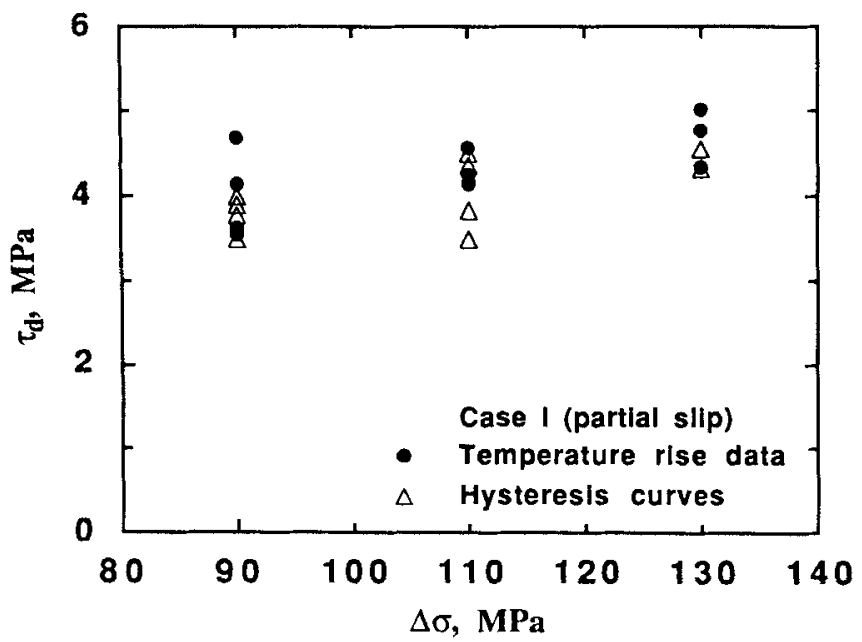

Fig. 7. Dynamic frictional shear stress, $\tau_{\mathrm{d}}$, estimated from Eq. (12). The shear stress was determined at a loading frequency of $25 \mathrm{~Hz}$. For comparison, both temperature rise data and the cyclic stress-strain (hysteresis) curves were used to determine the frictional energy dissipation. Both approaches provide similar results.

termining the theoretical stress-strain curves). Although fiber fracture was not included in the analysis of frictional energy dissipation, the total number of fractured fibers would be small for the loading histories examined. Thus, since the majority of interfacial slip zones would still be populated by

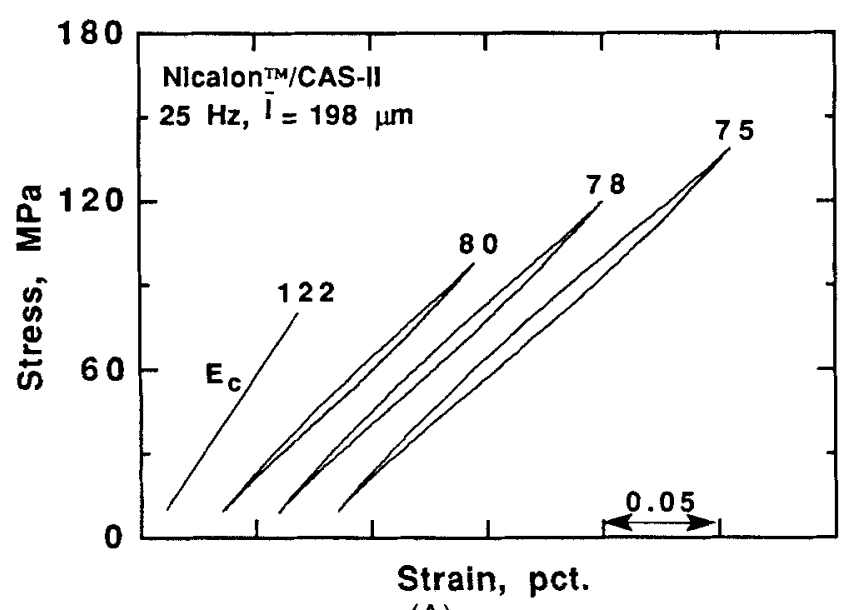

(A)

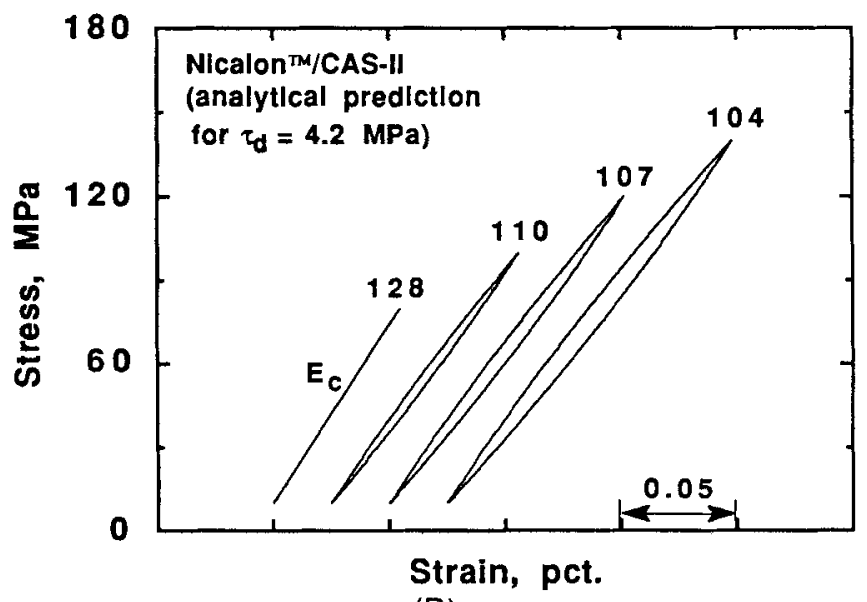

(B)

Fig. 8. Hysteresis curves formed during the cyclic loading of unidirectional Nicalon/CAS-II: (A) experimentally measured; (B) analytical prediction for the case of partial frictional slip. The average modulus for the fatigue cycle is shown above each curve (units: GPa). unfractured fibers, the error introduced in the calculation of shear stress by neglecting the slip of fractured fibers is expected to be small.

From the discussion presented above, the partial slip analysis is appropriate for determining the interfacial shear stress for the specific composite and loading conditions used in the frictional heating experiments; from Fig. 7, the dynamic shear stress is between approximately 3.5 and 5.0 MPa. Wang and Parvizi-Majidi ${ }^{17}$ have measured the frictional shear stress in Nicalon/CAS-II composites; the shear stress ranged from 13 to $18 \mathrm{MPa}$ (determined by fiber pushout and pushin experiments). These values correspond to the frictional shear stress which would be present in virgin material immediately after interfacial debonding. Under cyclic loading, however, the repeated frictional sliding of fibers (either fractured or unfractured) can produce wear damage along the fibermatrix interface. Thus the relatively low value of frictional shear stress $(3.5$ to $5.0 \mathrm{MPa}$ ) which was found in the present paper is most likely a consequence of interfacial wear damage that occurred during the fatigue experiments. Evidence for a reduction in frictional shear stress caused by the fatigue loading of composite materials has been obtained from fiber pushout experiments conducted by Mackin et al. ${ }^{8}$ on Ti-V composites. Singh and Sutcu ${ }^{18}$ have also observed a decrease in frictional shear during repeated fiber pushout/pushback experiments conducted with $\mathrm{SiC} /$ zircon composites.

\section{Summary}

A now technique for estimating the interfacial shear stress present during the cyclic loading of fiber-reinforced ceramics has been developed. To generalize the model, it will be necessary to include the influence of fractured fibers on the frictional energy dissipation which occurs during fatigue; this will require knowledge of the statistical distribution of fiber strength and the failure location of fibers. A potential advantage of the frictional heating technique for determining interfacial shear stress is that an average value of frictional shear stress is obtained, rather than a value based upon discrete measurements made on individual fiber/matrix interfaces. The technique can also be readily cxtended to allow determining the temperature dependence of interfacial shear stress. Also, since the temperature rise can be determined on a continuous basis, it is possible to continuously monitor the change in frictional shear stress which occurs during cyclic loading; this may be a useful approach for determining the influence of fiber coatings on the rate of interfacial wear. A limitation of the proposed frictional heating technique is that it can only provide information concerning the frictional shear stress which exists during cyclic loading. Other wellestablished approaches, such as fiber pushout ${ }^{1,2}$ and fiber pullout experiments, ${ }^{3,4}$ are required to determine the interfacial shear required to initiate debonding and the frictional shear stress which is present immediately after debonding.

\section{References}

'D. B. Marshall, "An Indentation Method for Measuring Matrix-Fiber Frictional Stresses in Fiber-Reinforced Ceramic Composites," J. Am. Ceram. Soc., 67 [12] C-259-C-260 (1984).

2D. B. Marshall and W. C. Oliver, "Measurement of Interfacial Mechanical Properties in Fiber-Reinforced Ceramic Composites," J. Am. Ceram. Soc., 70 [8] 542-48 (1987).

${ }^{3}$ K. T. Faber, S. H. Advani, J. K. Lee, and J.T. Jinn, "Frictional Stress Evaluation along the Fiber-Matrix Interface in Ceramic Matrix Composites," J. Am. Ceram. Soc., 69 [9] C-208-C-209 (1986).

${ }^{4}$ R.W. Goettler and K.T. Faber, "Interfacial Shear Stresses in SiC and $\mathrm{Al}_{2} \mathrm{O}_{3}$ Fiber-Reinforced Glasses," Ceram. Eng. Sci. Proc., 9 [7-8] 861-72 (1988).

s.W. Holmes and S. F. Shuler, "Temperature Rise During Fatigue of FibreReinforced Ccramics," J. Mater. Sci. Lett., 9 [11] 1290-91 (1990).

"J.W. Holmes and C. Cho, "Frictional Heating in a Fiber-Reinforced Ceramic Composite," J. Mater. Sci. Lett., in press.

${ }^{7} \mathrm{~J}$. W. Holmes and C. Cho, "Experimental Observations of Frictional Heating in Fiber-Reinforced Ceramics," J. Am. Ceram. Soc., in press. 
"T. J. Mackin, P. D. Warren, and A. G. Evans, "Fiber Pushout of a Fatigued Specimen"; presented at the 93d Annual Meeting of the American Ceramic Society, Cincinnati, OH, April 28-May 2, 1991 (Ceramic Matrix Composites Symposium, Paper No. 54-SV1-91).

${ }^{4} \mathrm{C}$. Cho, J.W. Holmes, and J. R. Barber, "Distribution of Matrix Cracks in a Uniaxial Ceramic Composite," J. Am. Ceram. Soc, in press.

${ }^{10}$ R. M. McMeeking and A.G. Evans, "Matrix Fatigue Cracking in Fiber Composite," Mech. Mater., 9, 217-27 (1990).

"B. N. Cox, "Interfacial Sliding Near a Fibrous or Layered Composite During Thermal Cycling," Acta Metall. Mater., 38 [12] 2411-24 (1990).

${ }^{12}$ M. D. Thouless and A. G. Evans, "Effects of Pull-Out on The Mechanical Properties of Ceramic Matrix Composites," Acta Metall, 36 [3] 517-22 (1988)

${ }^{13}$ M. D. Thouless, "A Re-Examination of the Analysis of Toughening in
Brittle-Matrix Composites," Acta Metall, 37 [9] 2297-304 (1989).

${ }^{14}$ R. A. Shimansky and H.T. Hahn, "Effect of Interfacial Properties on Matrix Cracking Stress of Fiber Reinforced Ceramics"; pp. 148-57 in Symposium on High Temperature Composites. Technomic Publishing Co., Lancaster, PA, 1989

15J. P. Holman, Heal Transfer; p. 346. McGraw-Hill, New York, 1986.

ro Information sheet on Nicalon fibers and calcium aluminosilicate provided by Corning Inc., Corning, N Y, 1991.

${ }^{17}$ S.W. Wang and A. Parvizi-Majidi, "Mechanical Behavior of Nicalon Fiber-Reinforced Calcium-Aluminosilicate Matrix Composites," Ceram. Eng. Sci. Proc., 11 [9-10] 1607-16 (1990).

${ }^{1}$ R. N. Singh and M. Sutcu, "Determination of Fiber-Matrix Interfacial Properties in Ceramic Matrix Composites by a Fiber Push-out Technique," J. Moter. Sci., 26, 2547-56 (1991). 